\title{
Diffractometer-Control Software For Bragg-Rod Measurements
}

\author{
Y. Yacoby $^{a}$, D.A. Walko ${ }^{b}$, D. Brewe ${ }^{c}$, M. Bretschneider ${ }^{a}$, M. Sowwan ${ }^{a}$, \\ R. Clarke ${ }^{d}$, R. Pindak ${ }^{e}$, and E.A. Stern ${ }^{c}$ \\ (a) Racah Institute of Physics, Hebrew University, Jerusalem, 91904 Israel \\ (b) MHATT-CAT, Argonne National Laboratory, Argonne, IL 60439 USA \\ (c) Department of Physics and PNC-CAT, University of Washington, Seattle, WA 98195 USA \\ (d) Department of Physics, University of Michigan, Ann Arbor, MI 48109 USA
}

(e) NSLS, Brookhaven National Laboratory, Upton, NY 11973 USA

\begin{abstract}
We present Generalized Diffractometer Control (GDC), a diffractometer-control software package developed specifically for high-precision measurements of Bragg rods; we discuss its features and analyze its performance in data collection. GDC, implemented at several APS beamlines, controls a six-circle diffractometer in either Eulerian or kappa geometry, yet does not assume a mechanically ideal diffractometer; instead, the measured directions of the diffractometer axes (and the direction of the incident beam) are input parameters. The Labview-based program features a graphical interface, making it straightforward to find all the commands and operations. Other features include optimized scans along Bragg rods, straightforward background subtraction, and extensive sets of pseudomotors.
\end{abstract}

\section{INTRODUCTION}

Accurate intensity measurements are critical to the success of surface and thin-film x-ray diffraction experiments. It has been an experimental challenge to accurately and efficiently measure the diffuse scattering due to the presence of a surface or interface (the so-called Bragg rods or crystal truncation rods). These rods, parallel to the surface normal, originate at Bragg points and can vary in intensity by several orders of magnitude. The need for accuracy is magnified in direct-method techniques, wherein the phase problem is typically addressed by oversampling the data along the Bragg rods. For example, in COherent Bragg-Rod Analysis (the COBRA method), the total scattering is broken into contributions from a known part and from an unknown part of the sample; the key to COBRA is that the phase of the unknown part varies slowly between adjacent data points along a Bragg rod $[1,2]$. Any errors in data collection limit the applicability of COBRA or any direct method. Therefore, we have developed a diffractometer-control software package, GDC or Generalized Diffractometer Control, optimized for high-precision measurement of Bragg rods. This Labview-based package has a graphical user interface (GUI) and offers many options not implemented in most commercial diffractometer software. In the following sections we describe the program's general layout and operation, focusing on its novel features, and its performance in the collection of Bragg-rod data.

\section{GENERAL PROGRAM DESCRIPTION AND NOVEL FEATURES}

GDC was written primarily in Labview, wherein an individual software routine is called a virtual instrument (VI); additional calculations are performed by Matlab routines. Communication to EPICS beamline control is via standard channel-access calls, although GDC can run on beamlines controlled by other systems. The GUI interface allows all available commands to be conveniently displayed for the user. Furthermore, nearly every step in the operation of GDC (not just data collected in scans) is saved to a text logfile. A display VI allows any scan to be viewed and printed, either graphically or numerically in a table.

CP705, Synchrotron Radiation Instrumentation: Eighth International Conference, edited by T. Warwick et al.

(C) 2004 American Institute of Physics 0-7354-0180-2/04/\$22.00 
The top-level VI, from which GDC starts, lists the VIs that perform all the tasks of the program. There are several sets of these VIs. One set consists of help files and reference tables, such as lists of motors, pseudomotors, and detectors. Another set consists of continuously running VIs, such as a detector display screen and a manual attenuator controller. The largest set of VIs controls operations such as moving, scanning, and calculating the sample orientation; only one of these can be called at a time. These operational VIs are described in some detail in a following section.

\section{Generalized Diffractometer Description}

GDC is designed to operate a six-circle goniometer with either kappa or Eulerian geometry. The axes are labeled for a kappa-style goniometer in Fig. 1a, and we will generally assume kappa geometry in this report. A number of global variables, such as information about the diffractometer, are stored in glob.vi; in these variables we find some of the novel features of the GDC system. One of the most conspicuous features of GDC is that it does not assume the diffractometer's rotation axes are aligned according to idealized specifications, as most diffraction calculations do [3]. Instead, a routine has been developed to measure the actual directions of the axes using an autocollimator on the detector arm and a mirror at the sample position, and only assumes that the ome (short for omega) axis is perpendicular to the nuи and psi axes. Such measurements have found goniometer axes deviating several mrad from ideal; these deviations are due partly to mechanical imperfections and partly to incorrect determination of the zeroposition of some axes. Furthermore, the incident x-ray beam can have an arbitrary direction that is, again, empirically determined. Not requiring the diffractometer to be square to the beam is a significant advantage for alignment of diffractometers on tables with limited degrees of freedom. To protect equipment from accidental collisions during operation, "cones of protection" are defined for the incident flightpath, the sample arm, and the detector arm. The vertices of these cones meet at the center of rotation and have an opening angle large enough to encompass the equipment they represent; to avoid collisions, the cones are not allowed to overlap, providing a more intelligent collision-protection system than merely defining upper and lower limits for each motor individually. Overlap of these cones is checked dynamically before each move.

Several other parameters are saved in glob.vi. These include the sample's "fixed axis," i.e., the reference direction of the sample when defining that the sample should point in a particular direction; the initial lattice parameters; and a list of the Bragg points used in defining the orientation matrix. Degeneracies from the various symmetry transformations of the diffractometer angles are also resolved here; the choice of angle combinations can be automatic (wherein the total angular motion of a given move is minimized) or manual.

\section{Detectors And Background Subtraction}

The detector arm used in COBRA experiments with GDC consists of guard slits, an XIA PF4 filter box, a "paddle," and a plastic scintillator and photomultiplier in current mode (to accurately handle high count rates). The paddle is a small aluminum arm with lead shielding on a stepper motor, rotating three different apertures into the beam as shown in Fig. 1b. A large aperture on the paddle is used only for initial alignment. The second aperture is a smaller opening that determines the resolution of the detector during data collection. The third aperture is a pair of openings offset to the side, with the central beam blocked; the purpose of these openings is to measure the background near a Bragg rod. Thus, Bragg-rod data and background are collected, point by point, with a simple rotation of this paddle; no diffractometer axes need move. This method of background subtraction is used because rocking curves at each point on a rod are not feasible at the data-point density required for COBRA. Long-term

plans call for replacing this paddle and detector assembly with a two-dimensional CCD detector, allowing data and background to be collected in a single frame.

\section{DIFFRACTOMETER OPERATION}

The main functionality of GDC is in the operational VIs. Several of these perform fairly straightforward functions, such as editing motor parameters, synchronizing the motor properties to the values in the EPICS motor record, measuring the background counts in detectors (when there are no x rays in the hutch), and entering comments into the log file. Other VIs are available to edit the list of Bragg points from which the reciprocal unit cell is calculated, using either three Bragg points to refine the orientation (or only two, in the case of cubic symmetry) or more than three to correct for misalignments (such as imperfect knowledge of the direction of the incident beam, or for a 

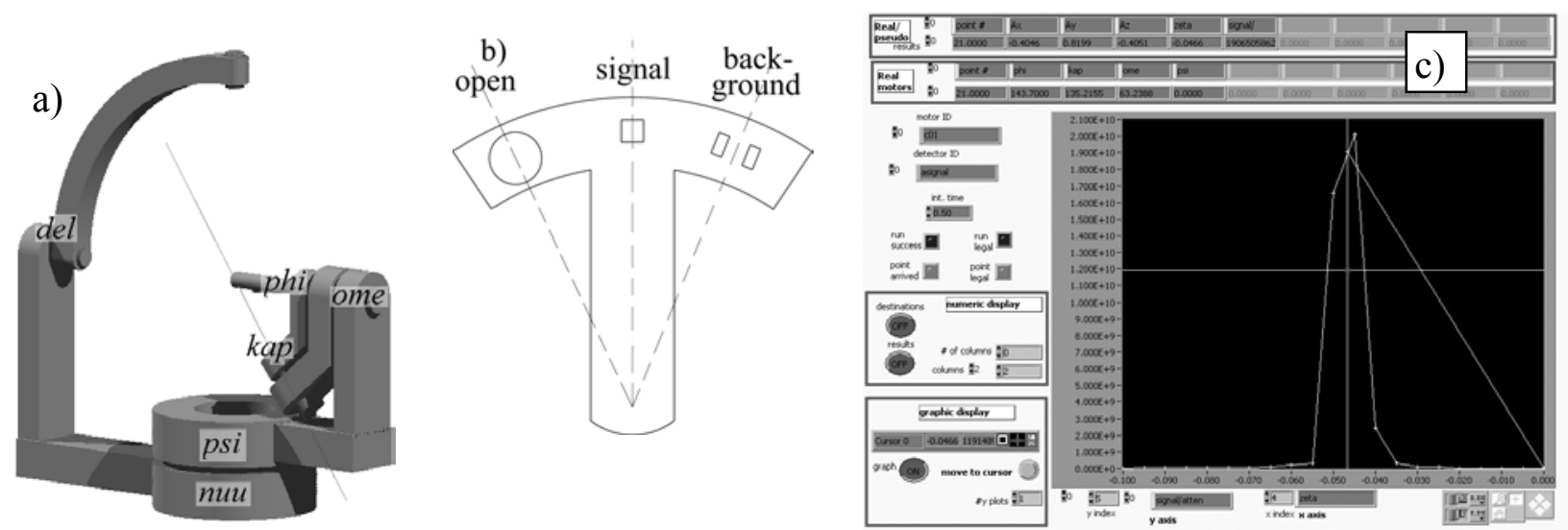

FIGURE 1. a) Schematic of kappa diffractometer with all axes at zero except $o m e=90^{\circ}$; the diagonal line symbolizes the incident beam direction. Note some atypical definitions: at $p s i$ and $n u u=0$, the $t$ th and ome axes are $45^{\circ}$ from the incident beam, chosen for convenience; and $t t h=0^{\circ}$ makes the detector point straight down, simplifying angle calculations when the detector is in this region. b) Schematic of the "paddle" showing its three positions. c) Screen shot of a lineup scan. While the scan was ongoing, the user moved the cursor to the peak and selected "move to cursor;" this point is also shown on the graph.

sample not exactly at the center of rotation). In the rest of this section, we describe the VIs for moving motors, operating detectors, scanning, tweaking motor positions, and aligning.

The VI for moving motors allows users to select a motor to move and enter a destination, while the current position of that motor is continuously updated. The functionality of GDC is greatly extended by an extensive set of "pseudomotors" and "combination motors." Pseudomotors are defined as having one-to-one correspondence with the real motors, while combination motors do not have this correspondence. A commonly used pseudomotor grouping consists of pseudomotors $\{\mathrm{h}, \mathrm{k}, \mathrm{l}, \mathrm{Xa}, \mathrm{Ya}, \mathrm{Za}\}$, with the real motors being the diffractometer axes; this set of pseudomotors allows a user to move to a given Bragg point with the sample's fixed axis in the [Xa,Ya,Za] direction. Alternately, a set of pseudomotors \{slit position, slit width\} can be defined for each slit when the real motors are the positions of the individual blades. An example set of combination motors is $\{\mathrm{Rx}, \mathrm{Ry}, \mathrm{Rz}$, zeta $\}$, which rotates the sample about vector $\mathbf{R}$ by angle zeta using the real motors \{phi,kap,ome,psi $\}$; a similar combination motor has been defined to rotate the detector about $\mathbf{R}$ with real motors $\{t t h, n u u\}$. Additional pseudomotors are straightforward to define in the Labview-based code.

The detectors have several operating modes. An automatic attenuator routine can insert or remove filters from the beam when the detector signal falls above or below the appropriate thresholds; this routine can operate during scans, so a single scan can collect data for a full Bragg rod, including the Bragg points. As described above, the three-position paddle determines the resolution of the detector and whether the central beam or the background is being observed. The detector mode can be set to differential, in which counts with the paddle at the background position are subtracted from counts with the paddle at the signal position. In fact, pseudo-detectors have been defined to compute the intensity normalized to the incident beam (monitored with an ion chamber) and to the strength of the attenuators being used with background counts and detector dark current subtracted.

Any set of real motors, pseudomotors, or combination motors can be scanned in GDC, and any detectors or pseudo-detectors can be plotted in the graphical display. A screen shot of a just-completed scan is shown in Fig. 1c. During a scan, the cursor can be placed at any point along the plot in order to move the motors there at the end of the scan. The VI for lining up is similar to scanning, except the input parameters include central position and motor range, rather than the starting and ending points of the scan. Macros have been defined to automatically prepare common steps of the alignment procedure, including scanning the detector transversely or longitudinally through a given Bragg point, scanning the sample through a given Bragg point, theta/two-theta scans, and rotating the sample and diffractometer to a new Bragg point.

The tweak VI also takes advantage of the GUI, doing more than allowing a motor, pseudomotor, or combination motor to be repeatedly moved by a given step size; the user can choose a detector whose value is plotted as a function of motor position. As with lineup scans, the cursor can be used to select a destination for the motor being tweaked once the optimal position is found.

Even though GDC can be used to align a sample with very high precision, it is not always possible to simply measure a Bragg rod by scanning along it. Therefore, an additional alignment step is used before performing a Bragg-rod scan. Cross-scans, which cut across the rod, are performed at several points along the rod; these scans are performed in on-the-fly mode to save time. Linear interpolation determines the empirical position of that rod in 
reciprocal space. From this information, a new set of correction pseudomotors, essentially, a redefinition of the $\{\mathrm{h}, \mathrm{k}, \mathrm{l}, \mathrm{Za}, \mathrm{Ya}, \mathrm{Za}\}$ pseudomotor set described above, is created for each rod. This significantly improves the ability to precisely scan along each rod.

\section{PERFORMANCE IN DATA COLLECTION}

To test the performance of GDC, we collected several symmetry-equivalent Bragg rods from a 16- $\AA$-thick film of $\mathrm{Gd}_{2} \mathrm{O}_{3}$ on a $\mathrm{GaAs}(100)$ substrate; this system exhibits $p 2$ symmetry. Two pairs of such rods, corrected for geometric effects including Lorentz and polarization factors, are shown in Fig. 2 and are nearly indistinguishable by eye. Signals near the anti-Bragg positions appear too weak to measure, but agreement across the rods is excellent. $R_{\text {int }}$, the "merging r-factor," is defined as $\sum\left|I-I_{\text {mean }}\right| / \sum I$ where the sums are over all reflections of intensity $I$ for which symmetry equivalents have been measured (with average intensity $I_{\text {mean }}$ ). The calculated value of $R_{\text {int }}$ from the four sets of symmetry-equivalent rods is a very low $3.8 \%$, with only points very near the Bragg peaks excluded. If the data points within $\sim 0.4 \AA^{-1}$ of the Bragg points are included, $R_{\text {int }}$ jumps to $15 \%$; measurements near the highintensity bulk Bragg points are obviously very sensitive to alignment compared to measurements along the onedimensional Bragg rods. This test confirms the high precision of GDC in collecting Bragg rod data; improvements to optimize data collection at Bragg points are, of course, possible in this flexible software.
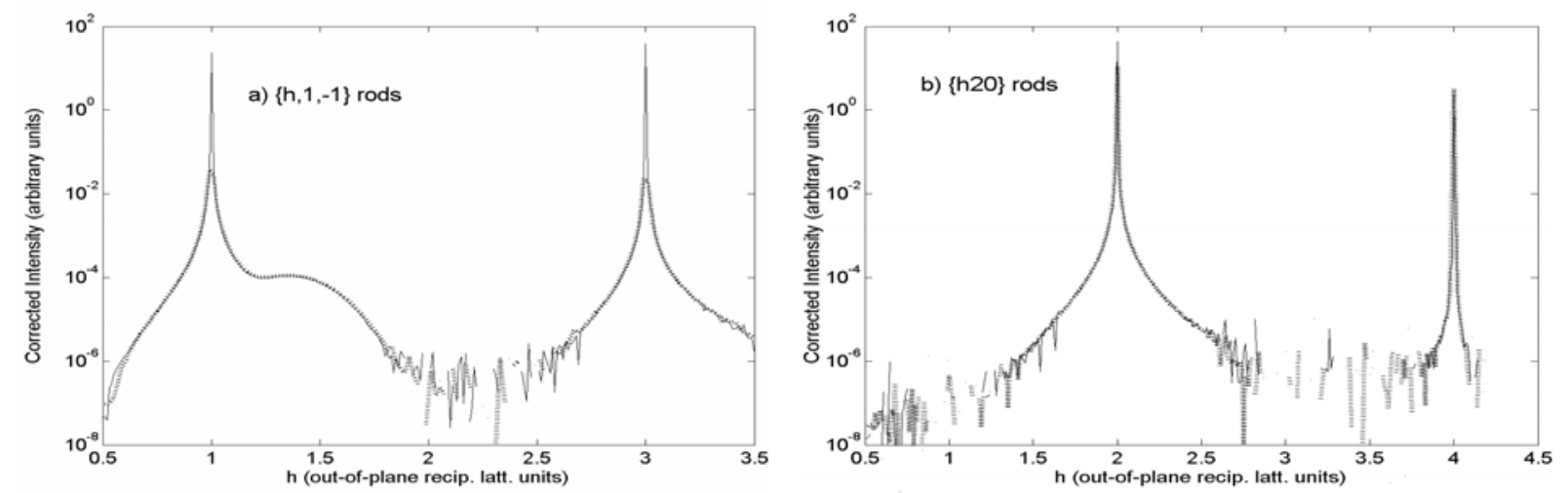

FIGURE 2. Symmetry-equivalent pairs of Bragg rods collected on a $\mathrm{Gd}_{2} \mathrm{O}_{3} / \mathrm{GaAs}(100)$ sample with GDC. $h$ is the reciprocallattice unit in the direction of the surface normal. a) Solid line: $(h 1 \overline{1})$ rod, dotted line: $(h \overline{1} 1)$ rod. b) Solid line: $(h 20)$ rod, dotted line: $(h \overline{2} 0)$ rod.

\section{ACKNOWLEDGMENTS}

We thank R. MacHarrie, J. Pitney, E.M. Dufresne, J.O. Cross, and C. Cionca for experimental assistance in COBRA measurements. This project was supported by the US-Israel Bi-National Science Foundation under Contract No. 1999-187. Work at the University of Washington and PNC-CAT was supported by Department of Energy Grants No. DE-FG03-98ER45681 and DE-FG03-97ER45628. Work at MHATT-CAT was supported by the U.S. Department of Energy, Grant No. FG02-03ER46023. Use of the APS was supported by the U.S. Department of Energy, Basic Energy Sciences, Office of Energy Research, under Contract No. W-31-109-Eng-38.

\section{REFERENCES}

1. Yacoby, Y., Sowwan, M., Stern, E., Cross, J.O., Brewe, D., Pindak, R., Pitney, J., Dufresne, E.M., and Clarke, R., Nature Materials 1, 99-101 (2002).

2. Sowwan, M., Yacoby, Y., Pitney, J., MacHarrie, R., M. Hong, M., Cross, J., Walko, D.A., Clarke, R., and Stern, E.A., Phys. Rev. B 66, 205311 (2002).

3. Busing, W.R., and Levy, H.A., Acta Cryst. 22, 457-464 (1967). 